Lorenz Dominance and the Variance of Logarithms

Author(s): James E. Foster and Efe A. Ok

Source: Econometrica, Vol. 67, No. 4, (Jul., 1999), pp. 901-907

Published by: The Econometric Society

Stable URL: http://www.jstor.org/stable/2999462

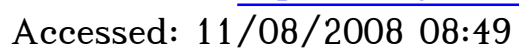

Your use of the JSTOR archive indicates your acceptance of JSTOR's Terms and Conditions of Use, available at http://www.jstor.org/page/info/about/policies/terms.jsp. JSTOR's Terms and Conditions of Use provides, in part, that unless you have obtained prior permission, you may not download an entire issue of a journal or multiple copies of articles, and you may use content in the JSTOR archive only for your personal, non-commercial use.

Please contact the publisher regarding any further use of this work. Publisher contact information may be obtained at http://www.jstor.org/action/showPublisher?publisherCode=econosoc.

Each copy of any part of a JSTOR transmission must contain the same copyright notice that appears on the screen or printed page of such transmission.

JSTOR is a not-for-profit organization founded in 1995 to build trusted digital archives for scholarship. We work with the scholarly community to preserve their work and the materials they rely upon, and to build a common research platform that promotes the discovery and use of these resources. For more information about JSTOR, please contact support@jstor.org. 


\title{
LORENZ DOMINANCE AND THE VARIANCE OF LOGARITHMS
}

\author{
By James E. Foster And EFe A. OK ${ }^{1}$
}

\section{INTRODUCTION}

THE VARIANCE OF LOGARITHMS $V_{L}$ is a widely used measure of dispersion, owing in part to its natural link with wage determination models, its useful decomposition formula, and its special relationship with the lognormal distribution. ${ }^{2}$ At the same time there is a longstanding view that $V_{L}$ is not a proper measure of inequality due to its clear violation of Dalton's (1920) "principle of transfers" and its consequent disregard for the Lorenz criterion-a ranking generally accepted as the unambiguous arbiter of inequality comparisons. ${ }^{3}$ This criticism of $V_{L}$ is often dismissed on the grounds that problems can only occur at the extreme upper tail of the distribution. Cowell (1977), for example, has proved that a marginal transfer from poor to rich can decrease $V_{L}$ (hence violating the transfer principle) only if the higher income is greater than $e$ times the geometric mean of the distribution in question. This is commonly viewed as suggesting that when $V_{L}$ violates the Lorenz criterion, the Lorenz curves can differ only slightly from one another in a very limited range of the "richest" population.

In this note we offer a strikingly different picture of the potential conflict between the variance of logarithms and the Lorenz criterion. In particular, we find that disagreements between $V_{L}$ and the Lorenz ranking may involve broad-based changes in incomes up and down the distribution (not just in the extreme upper tail). Moreover, they can involve comparisons in which the Lorenz curves are widely divergent from one another. Our main result shows that the following "worst-case scenario" can occur: One distribution, whose Lorenz curve approximates the $45^{\circ}$ line of equality, Lorenz dominates a second distribution, whose Lorenz curve approximates the $L$-shaped curve of complete inequality, and yet $V_{L}$ concludes that the second is more equal than the first. Consequently, the variance of logarithms is capable of making very serious errors.

\section{DEFINITIONS}

An income distribution is a positive real vector $\mathbf{x}=\left(x_{1}, \ldots, x_{n}\right)$, where $n$ is its population size and $x_{i}$ is the income of person $i$. Associated with $\mathbf{x}$ is a distribution

\footnotetext{
${ }^{1}$ We would like to thank Roland Benabou, Kin Blackburn, Peter Lambert, Bob Margo, a co-editor and an anonymous referee for helpful comments, and Artyom Shneyerov for his superb research assistance. This paper was written while Foster was a visitor in the Department of Economics at New York University. He thanks the Department for its hospitality. Support from the C. V. Starr Center for Applied Economics at New York University and from the John D. and Catherine T. MacArthur Foundation (through its Network on Inequality and Poverty in Broader Perspective) is gratefully acknowledged.

${ }^{2}$ See Foster and Sen (1997). $V_{L}$ figures prominently in the recent literature on U.S. wage inequality (e.g., Karoly (1992), Levy and Murnane (1992), Juhn, Murphy, and Pierce (1993), and Card and Lemieux (1994)), discussions of regional ( $\sigma$-)convergence (e.g., Sala-i-Martin (1996)), and self-selection models of inequality (e.g., Heckman and Honore (1990)).

${ }^{3}$ See, for example, Atkinson (1970), Cowell (1977), and Sen (1997).
} 
function $F_{\mathbf{x}}$, which, for each $s$, indicates the share $F_{\mathbf{x}}(s)$ of the population receiving an income of $s$ or below. The Lorenz curve $L_{\mathbf{x}}$ gives the cumulative share of income $L_{\mathbf{x}}(p)$ held by the poorest cumulative share $p$ of the population. Noting that $F_{\mathbf{x}}^{-1}(p)$ is the income of the person whose position in the distribution is $p$, we see that

$$
L_{\mathbf{x}}(p) \equiv \frac{1}{\mu_{\mathbf{x}}} \int_{0}^{p} F_{\mathbf{x}}^{-1}(t) d t, \quad 0 \leq p \leq 1,
$$

defines the Lorenz curve of the distribution $\mathbf{x}$, where $\mu_{\mathbf{x}}$ is the mean of the distribution. ${ }^{4}$ We say that $\mathbf{x}$ Lorenz dominates $\mathbf{y}$, denoted by $\mathbf{x} L \mathbf{y}$, whenever $L_{\mathbf{x}}(p) \geq L_{\mathbf{y}}(p)$ holds for all $p \in[0,1]$, with strict inequality for some $p .^{5}$

An inequality measure $I$ is a mapping from the set of all income distributions of arbitrary population size to the reals, where $I(\mathbf{x})$ denotes the level of inequality associated with the distribution $\mathbf{x}$. We say that $I$ is Lorenz consistent if $\mathbf{x} L \mathbf{y}$ implies that $I(\mathbf{x})<$ $I(\mathbf{y})$ for any two distributions $\mathbf{x}$ and $\mathbf{y}$. Two commonly used inequality measures, the Gini coefficient and Theil's second measure, are defined as $G(\mathbf{x}) \equiv\left(1 /\left(2 n^{2} \mu_{\mathbf{x}}\right) \sum_{k=1}^{n} \sum_{l=1}^{n}\left|x_{k}-x_{l}\right|\right.$ and $T_{2}(\mathbf{x}) \equiv \ln \mu_{\mathbf{x}}-\ln g_{\mathbf{x}}$, where $g_{\mathbf{x}} \equiv\left(\prod_{k=1}^{n} x_{k}\right)^{1 / n}$ is the geometric mean of $\mathbf{x}$. It is readily observed that the inequality measures $G$ and $T_{2}$ are Lorenz consistent.

The variance of logarithms, written as $V_{L}$, is defined as the variance applied to the distribution of $\log$ incomes. In other words, where $\ln \mathbf{x} \equiv\left(\ln x_{1}, \ldots, \ln x_{n}\right)$, we have $V_{L}(\mathbf{x}) \equiv(1 / n) \sum_{k=1}^{n}\left(\ln x_{k}-\mu_{\ln \mathbf{x}}\right)^{2}$. Since $\mu_{\ln \mathbf{x}}=\ln g_{\mathbf{x}}$, letting $f(s, g) \equiv(\ln s-\ln g)^{2}$, we may write

$$
V_{L}(\mathbf{x})=\frac{1}{n} \sum_{k=1}^{n} f\left(x_{k}, g_{\mathbf{x}}\right) .
$$

Unlike the previous two measures, $V_{L}^{\circ}$ is not Lorenz consistent.

\section{THE EXTENT OF LORENZ INCONSISTENCY}

We are interested in identifying situations where $V_{L}$ violates Lorenz consistency, that is, $\mathbf{x} L \mathbf{y}$ and yet $V_{L}(\mathbf{x})>V_{L}(\mathbf{y})$. Our starting point is the single transfer case. Let $\mathbf{y}(\delta)$ be the distribution obtained from $\mathbf{y}$ by a transfer of size $\delta>0$ from a richer individual (say person $h$ ) to a poorer individual (say person $l$ ). A violation of Lorenz consistency for $\mathbf{x}=\mathbf{y}(\delta)$ and $\mathbf{y}$ then corresponds to a violation of the transfer principle - a topic that has been studied by Cowell (1977, p. 163) for a marginal transfer in which $\delta$ is arbitrarily small. Indeed,

$$
\left.\frac{d V_{L}(\mathbf{y}(\delta))}{d \delta}\right|_{\delta=0}=\frac{1}{n}\left(f_{1}\left(y_{l}, g_{\mathbf{y}}\right)-f_{1}\left(y_{h}, g_{\mathbf{y}}\right)\right)
$$

describes the effect of a marginal progressive transfer on $V_{L}$. It is easily verified that $f\left(\cdot, g_{\mathbf{y}}\right)$ is decreasing on $\left(0, g_{\mathbf{y}}\right]$ and increasing on $\left[g_{\mathbf{y}}, \infty\right)$, while it is convex on $\left(0, e g_{\mathbf{y}}\right]$ and

${ }^{4}$ Formally, $F_{\mathbf{x}}^{-1}(p) \equiv \inf _{s \geq 0}\left\{F_{\mathbf{x}}(s) \geq p\right\}$ is the generalized inverse of $F_{\mathbf{x}}$ (Gastwirth (1971)) and the Lorenz curve of $\mathbf{x}$ is the graph of the function $L_{\mathbf{x}}$.

${ }^{5}$ It is well known that, where $\mathbf{x}$ and $\mathbf{y}$ have identical population sizes and means, $\mathbf{x} L \mathbf{y}$ holds if and only if $\mathbf{x}$ can be obtained from $\mathbf{y}$ by a finite number of progressive transfers (from richer to poorer individuals); see, for instance, Dasgupta, et al. (1973) and Foster (1985). This is one of the reasons why Lorenz dominance is generally accepted as an unambiguous method of making ordinal inequality comparisons. 


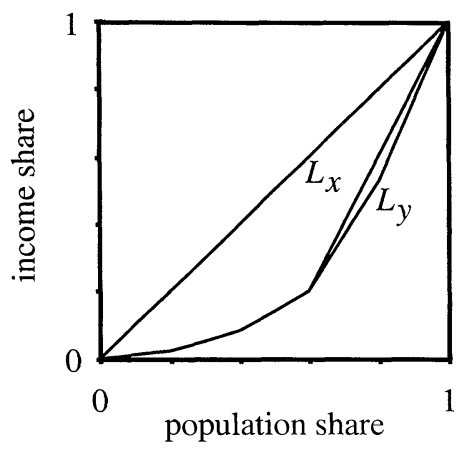

FIGURE 1.-Lorenz inconsistent example.

concave on $\left[e g_{\mathbf{y}}, \infty\right)$. Consequently, $y_{l} \geq e g_{\mathrm{y}}$ is a sufficient condition for a violation to occur, while $y_{l}>g_{\mathrm{y}}$ and $y_{h} \geq e g_{\mathrm{y}}$ are necessary. This observation leaves one with the impression that $V_{L}$ will go against the transfer principle only when the associated transfer takes place in the extreme upper tail of the distribution, and hence that the extent of the disagreement of $V_{L}$ with the Lorenz criterion is likely to be insignificant.

As an illustration, consider the distribution $\mathbf{y}=(2,5,10,28,40)$. Since $g_{\mathbf{y}}=10.229>10$ and $e g_{\mathbf{y}}=27.721<28$, the only possible violation of the transfer principle due to a marginal transfer involves the final two incomes. Indeed, $f_{1}\left(y_{4}, g_{\mathrm{y}}\right)=0.071>0.068=$ $f_{1}\left(y_{5}, g_{\mathbf{y}}\right)$, and thus a marginal transfer from person 5 to person 4 increases $V_{L}$. Now to what extent can the Lorenz curve be altered by a progressive transfer that simultaneously raises $V_{L}$ ? The maximal upward shift is obtained by choosing $\mathbf{x}=\mathbf{y}(6)=(2,5,10,34,34)$ in which the incomes of persons 4 and 5 are equalized. In this case, $V_{L}$ rises slightly (from 1.2100 to 1.2124 ) while, as depicted in Figure 1, the resulting upward shift in the Lorenz curve is modest with only a slight change of 0.028 in the Gini coefficient.

The picture changes appreciably when we go beyond the single transfer case. Consider, for example, the distributions

$$
\mathbf{x}=(1,1,1,41,41,41,41,41,41,41) \quad \text { and } \quad \mathbf{y}=(1,1,1,1,1,1,1,1,1,281) .
$$

Clearly, $\mathbf{x}$ is obtained from $\mathbf{y}$ by means of six progressive transfers. Figure 2 shows that the Lorenz dominance of $\mathbf{x}$ over $\mathbf{y}$ is broad-based, as the curves depart for $70 \%$ of the

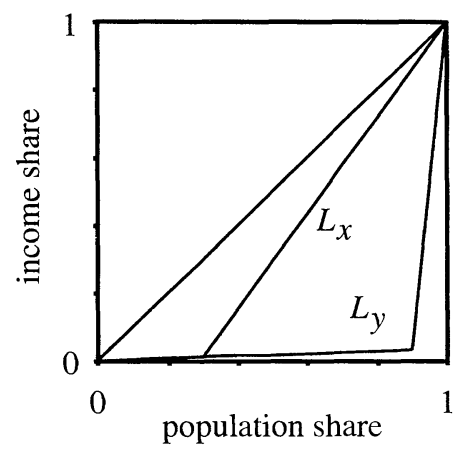

FIGURE 2.-Extreme Lorenz inconsistency. 
population, and quite extensive since the area between them is very large. Moreover, the Gini coefficient and Theil's second measure strongly support the judgment of the Lorenz ranking. ${ }^{6}$ The Gini coefficient, in particular, rises by almost $200 \%$ indicating that the area between the line of perfect equality and $L_{\mathbf{y}}$ is three times larger than the area between the line of perfect equality and $L_{\mathbf{x}}$. Yet, $V_{L}(\mathbf{x})=2.90>2.86=V_{L}(\mathbf{y})$, that is, $V_{L}$ declares $\mathbf{x}$ to be more unequal than $\mathbf{y}$ ! The Lorenz inconsistency of $V_{L}$ can be very significant indeed.

The logic behind this example can be extended to obtain the following surprising result.

Proposition: For any $\epsilon>0$, there exist two income distributions $\mathbf{x}$ and $\mathbf{y}$ satisfying $G(\mathbf{x})<\epsilon$ and $G(\mathbf{y})>1-\epsilon$ for which $\mathbf{x} L \mathbf{y}$ and $V_{L}(\mathbf{x})>V_{L}(\mathbf{y})$.

PROOF: For any integers $n \geq 3$ and $m \geq 1$ with $m<n$, define

$$
\mathbf{x}^{m, n} \equiv(\underbrace{1, \ldots, 1}_{m \text { times }}, \underbrace{\theta, \ldots, \theta}_{n-m \text { times }}),
$$

where $\theta \equiv(n B /(n-m))+1$ for an arbitrary $B>0$. By direct computation, we find

$$
\begin{aligned}
G\left(\mathbf{x}^{m, n}\right) & =\frac{B}{B+1}\left(\frac{m}{n}\right) \text { and } \\
V_{L}\left(\mathbf{x}^{m, n}\right) & =\frac{m}{n}\left(1-\frac{m}{n}\right)\left(\ln \left(\frac{B}{1-(m / n)}+1\right)\right)^{2} .
\end{aligned}
$$

Define

$$
\varphi(\alpha, B) \equiv \alpha(1-\alpha)\left(\ln \left(\frac{B}{1-\alpha}+1\right)\right)^{2} \text { for all } \alpha \in[0,1) \text { and } B>0 .
$$

Four observations about the function $\varphi(\alpha, B)$ are in order. First, notice that $\varphi$ can be continuously extended to $[0,1]$ by using l'Hôpital's rule and setting $\varphi(1, B)=0$ for all $B>0$. Second, we have

$$
\varphi(\alpha, B)<\varphi(1-\alpha, B) \text { for all } \alpha \in(0,1 / 2) \text { and } B>0
$$

as can be readily verified. Third,

$$
\frac{\partial \varphi(\alpha, B)}{\partial \alpha}=\left((1-2 \alpha) \ln \left(\frac{B}{1-\alpha}+1\right)+\frac{2 \alpha B}{B+(1-\alpha)}\right) \ln \left(\frac{B}{1-\alpha}+1\right)
$$

so that $\varphi$ is a strictly increasing function of $\alpha$ on [0,1/2], given any $B>0$. Finally, it is clear that for any $\bar{\alpha}>1 / 2$, there exists a $\bar{B}>0$ large enough to guarantee that $(\partial \varphi(\alpha, B) / \partial \alpha)<0$ for all $\alpha \geq \bar{\alpha}$ and $B \geq \bar{B}$.

Now pick any $\epsilon>0$, and choose any $\alpha_{1} \in(0, \min \{\epsilon, 1 / 2\})$. By using the last observation on $\varphi$ noted above, we may find a $B_{1}>0$ such that

$$
\varphi(\cdot, B) \text { is strictly decreasing on }\left[1-\alpha_{1}, 1\right] \text { for all } B \geq B_{1} .
$$

${ }^{6}$ Here we have $G(\mathbf{x})=.29, G(\mathbf{y})=.87, T_{2}(\mathbf{x})=.77$, and $T_{2}(\mathbf{y})=2.80$. 
But then, using the intermediate value theorem, we conclude that there must exist an $\alpha_{1}^{*}(B) \in(1 / 2,1)$ such that

$$
\varphi\left(\alpha_{1}, B\right)=\varphi\left(\alpha_{1}^{*}(B), B\right) \text { for all } B \geq B_{1} .
$$

Moreover, by (4) and (5), we must have $1-\alpha_{1}<\alpha_{1}^{*}(B)$ for all $B \geq B_{1}$. Now take any $\gamma<\epsilon$ and define

$$
\alpha_{2}(B) \equiv \max \left\{\alpha_{1}^{*}(B)+\frac{1}{2}\left(1-\alpha_{1}^{*}(B)\right),(1-\epsilon)+\gamma\right\} \text { for all } B>0 .
$$

Clearly, $1>\alpha_{2}(B)>\alpha_{1}^{*}(B)>1-\alpha_{1}>\alpha_{1}$ (for $B \geq B_{1}$ ) so that, by (5), we have

$$
\varphi\left(\alpha_{2}(B), B\right)<\varphi\left(\alpha_{1}^{*}(B), B\right) \text { for all } B \geq B_{1} .
$$

In addition, by the definition of $\alpha_{2}(B)$, there must exist $B_{2}>0$ such that

$$
\frac{B}{B+1} \alpha_{2}(B) \geq \frac{B}{B+1}((1-\epsilon)+\gamma)>1-\epsilon \text { for all } B \geq B_{2} .
$$

Now let $\hat{B}=\max \left\{B_{1}, B_{2}\right\}$ and notice that $\alpha_{1}<\alpha_{2}(\hat{B})$. Consequently, by denseness of the rationals in the reals, we may choose integers $n, m_{1}$, and $m_{2}$ such that $m_{1}<m_{2}$, $m_{1} / n \approx \alpha_{1}$, and $m_{2} / n \approx \alpha_{2}(\hat{B})$. But then, letting $\mathbf{x} \equiv \mathbf{x}^{m_{1}, n}$ and $\mathbf{y} \equiv \mathbf{x}^{m_{2}, n}$, we obtain

$$
G(\mathbf{x}) \approx \frac{\hat{B}}{\hat{B}+1} \alpha_{1}<\epsilon \text { and } G(\mathbf{y}) \approx \frac{\hat{B}}{\hat{B}+1} \alpha_{2}(\hat{B})>1-\epsilon
$$

by (3), the definition of $\alpha_{1}$, and (7). Moreover, (3) and (6) yield that $V_{L}(\mathbf{y}) \approx \varphi\left(\alpha_{2}(\hat{B}), \hat{B}\right)$ $<\varphi\left(\alpha_{1}^{*}(\hat{B}), \hat{B}\right)=\varphi\left(\alpha_{1}, \hat{B}\right) \approx V_{L}(\mathbf{x})$. Finally, $\mathbf{x} L \mathbf{y}$ follows from $m_{1}<m_{2}$ by construction.

Q.E.D.

Thus, the variance of logarithms is not only Lorenz inconsistent, but it can disagree with the Lorenz criterion in such a radical way that it misranks two distributions that are deemed to be almost perfectly equal and almost perfectly unequal by the Gini index. ${ }^{7}$ We thus conclude that there is essentially no limit to the disagreement between $V_{L}$ and the Lorenz ordering of income distributions.

Why does $V_{L}$ have this unfortunate characteristic? In other words, what is the driving force behind the above proposition? To answer this, let us return to the example depicted in Figure 2. The geometric mean rises in going from $\mathbf{y}$ to $\mathbf{x}$, which is only natural given the fact that the geometric mean is a Lorenz consistent equality measure for comparing distributions with fixed average income. Now observe that

$$
\begin{aligned}
V_{L}(\mathbf{x})-V_{L}(\mathbf{y})= & \frac{1}{10} \sum_{k=1}^{10}\left(f\left(x_{k}, g_{\mathbf{y}}\right)-f\left(y_{k}, g_{\mathbf{y}}\right)\right) \\
& +\frac{1}{10} \sum_{k=1}^{10}\left(f\left(x_{k}, g_{\mathbf{x}}\right)-f\left(x_{k}, g_{\mathbf{y}}\right)\right) \\
= & (7.01-2.86)+(2.90-7.01) .
\end{aligned}
$$

${ }^{7}$ In other words, the area between the two Lorenz curves is arbitrarily close to its maximum displacement of $1 / 2$. The use of the Gini index here is natural, but not essential. For instance, we can adapt the proof of the proposition to show the following result for Theil's second measure: For any $\epsilon>0$, there exist two income distributions $\mathbf{x}$ and $\mathbf{y}$ satisfying $T_{2}(\mathbf{x})<\epsilon$ and $T_{2}(\mathbf{y})>1 / \epsilon$ for which $\mathbf{x} L \mathbf{y}$ and $V_{L}(\mathbf{x})>V_{L}(\mathbf{y})$. 
This decomposition clearly shows that while the constituent transfers decrease the variance of log incomes evaluated at the original geometric mean, the resultant change in $g_{\mathbf{y}}$ raises $V_{L}$ back above its original level. In other words, the direct effect the transfers have on the income levels of the individuals is here outweighed by their indirect effect via the geometric mean. Consequently, this example points to the variation of the geometric mean as a main culprit in the massive disagreement between $V_{L}$ and the Lorenz criterion. $^{8}$

\section{CONCLUDING COMMENTS}

The Lorenz criterion is generally recognized as the fundamental tool for making inequality comparisons. When Lorenz curves cross, the criterion has nothing to say, and Lorenz-consistent indices-including the Gini coefficient, the coefficient of variation, and the Theil measures - can disagree. When Lorenz curves are unambiguously ranked so that the criterion applies, all of these standard indices will unerringly follow its judgment. While it has long been recognized that the variation of logarithms may go against the Lorenz criterion, the specific cases where this occurs were thought to involve relatively minor disagreements, with Lorenz curves departing slightly at the upper incomes. Our main result shows that this view is incorrect-that the variance of logarithms can err in even the most extreme examples of Lorenz-comparable distributions. The notion of dispersion captured by variance of logarithms can dramatically depart from the notion of "inequality" as traditionally defined.

How likely are such errors to arise in practice? On this question there are as yet no definitive answers. Creedy (1977) is often cited as suggesting that problems from using $V_{L}$ are highly unlikely. ${ }^{9}$ However, his analysis only considers the case of a single marginal transfer; and since Lorenz comparisoňs typically involve a series of discrete transfers (and a changing geometric mean), these results are not directly relevant. Moreover, even if one were interested in the likelihood that a marginal transfer between two incomes drawn at random will violate the transfer principle, the estimate given by Creedy is actually an order of magnitude too low. We have shown elsewhere (Foster and Ok (1997)) that when incomes are drawn directly from microdata or from standard statistical distributions, such as the lognormal, Singh-Maddala and beta-II, fitted to US data (cf. Singh and Maddala (1976) and McDonald (1984)) the likelihood of a violation is somewhere between $8 \%$ to $12 \% .^{10}$ The probability that a single marginal transfer will generate a Lorenz inconsistency for $V_{L}$ is not easily dismissed as negligible.

\footnotetext{
${ }^{8}$ Therefore, such large disagreements might be avoided if the variation in the geometric mean stays within certain bounds. Given the definition of Theil's second measure as $T_{2}(\mathbf{x})=\ln \mu_{\mathbf{x}}-\ln g_{\mathbf{x}}$, one may thus benefit from using $V_{L}$ in conjunction with $T_{2}$ in practice.

${ }^{9}$ See, for instance, Heckman and Honore (1990, footnote 6).

${ }^{10}$ The methodology behind these estimates can be briefly described as follows. Suppose that two incomes, $s$ and $t$, are chosen at random from a probability distribution with a cdf $F$ and a geometric mean $g$. Let $\pi$ denote the probability of the event $\left\{\Delta V_{L} \gtrless 0\right.$ and $\left.s \gtrless t\right\}$ conditional on a small transfer taking place from $s$ to $t$. By (2) and the discussion that follows it, $\pi$ must be the probability of the event $\{\min \{s, t\}>e g\} \cup\left\{s \in(g, e g), t>e g, f_{1}(s, g)>f_{1}(t, g)\right\} \cup\left\{t \in(g, e g), s>e g, f_{1}(s, g)<\right.$ $\left.f_{1}(t, g)\right\}$. But then we have
}

$$
\pi=(1-F(e g))^{2}+2 \int_{g}^{e g}(1-F(h(\omega))) d F(\omega)
$$

where $h:(g, e g) \rightarrow(e g, \infty)$ is defined via $f_{1}(h(\omega), g)=f_{1}(\omega, g)$. Estimates are then obtained from this formula using a standard numerical approximation method. 
Finally, we note that even when an outright conflict is not observed, there is significant scope for the phenomenon to affect the cardinal values of $V_{L}$, thereby altering perceptions and explanations of distributional changes. For example, a significant general compression of incomes may instead be interpreted as a minor compression if the variance of logarithms misreads the smoothing at the top as an increase in inequality. This built-in possibility for error would thus raise questions about analyses based exclusively on $V_{L}$. Future studies should provide evidence of the robustness of their conclusions by employing one or more of the standard, well-behaved inequality measures in addition to the variance of logarithms.

Dept. of Economics, Vanderbilt University, Nashville, TN 37235, U.S.A.; fosterje@ctrvax.vanderbilt.edu,

$$
\text { and }
$$

Dept. of Economics, New York University, 269 Mercer St., New York, NY 10003, U.S.A.; okefe@fasecon.econ.nyu.edu.

Manuscript received June, 1997; final revision received June, 1998.

\section{REFERENCES}

AtKinson, A. B. (1970): "On the Measurement of Inequality," Journal of Economic Theory, 2, 244-263.

CARD, D., AND T. LEMIEUX (1994): "Changing Wage Structure and Black-White Wage Differentials," American Economic Review, 84, 29-33.

Cowell, F. A. (1977): Measuring Inequality. New York: Wiley.

Creedy, J: (1977): "The Principle of Transfers and the Variance of Logarithms," Oxford Bulletin of Economics and Statistics, 39, 152-158.

Dalton, H. (1920): "The Measurement of Inequality of Incomes," Economic Journal, 30, 348-361.

Dasgupta, P., A. K. Sen, And D. Starrett (1973): "Notes on the Measurement of Inequality," Journal of Economic Theory, 6, 180-187.

Foster, J. E. (1985): “Inequality Measurement," in Fair Allocation, ed. by H. P. Young. Providence: American Mathematical Society Proceedings in Applied Mathematics, Vol. 33.

Foster, J. E., AND E. A. OK (1997): "Lorenz Dominance and the Variance of Logarithms," C. V. Starr Center Working Paper 97-25, New York University.

Foster, J. E., AND A. SEN (1997): “On Economic Inequality After a Quarter Century," in On Economic Inequality, by A. Sen. Oxford: Clarendon Press.

GASTwiRTH, J. L. (1971): “A General Definition of the Lorenz Curve," Econometrica, 39, 1037-1039.

Heckman, J. J., AND B. Honore (1990): "The Empirical Content of the Roy Model," Econometrica, $58,1121-1150$.

Juhn, C., K. M. Murphy, And B. Pierce (1993): "Wage Inequality and the Rise in Returns to Skill," Journal of Political Economy, 101, 410-442.

KAROLY, L. A. (1992): "Changes in the Distribution of Individual Earnings in the United States: 1967-1986," Review of Economics and Statistics, 74, 107-115.

LEVY, F., AND R. J. MURNANE (1992): "U.S. Earnings Levels and Earnings Inequality: A Review of Recent Trends and Proposed Explanations," Journal of Economic Literature, 30, 1333-1381.

MCDonAld, J. B. (1984): "Some Generalized Functions for the Size Distribution of Income," Econometrica, 52, 647-664.

Sala-I-Martin, X. (1996): "Regional Cohesion: Evidence and Theories of Regional Growth and Convergence," European Economic Review, 40, 1325-1352.

SEN, A. K. (1997): On Economic Inequality, Expanded Edition. Oxford: Clarendon Press.

Singh, S. H., AND G. S. Maddala (1976): "A Function for Size Distribution of Incomes," Econometrica, 44, 963-970. 